craniotomy surgeries followed by adjuvant treatments (2005-2014) were derived from the National Cancer Database (NCDB). The time intervals (days) from the date of diagnosis to the initiation date of adjuvant treatment [radiation therapy only (RT), chemotherapy only, concurrent chemoradiation (CRT), or non-concurrent RT and chemotherapy] were categorized into quartiles (Q1-Q4). Kaplan-Meier method and Cox proportional hazards regression were applied for survival analysis. Multivariate logistic regression was performed to compare differences in treatment timing, intervention modalities, and secondary outcomes. The patients underwent biopsy obtained significant survival benefit by having delayed adjuvant treatment [comparing to Q1, Q2: HR (hazard ratio), 0.88, Q3: HR, 0.86]. For patients underwent resection, the prolonged waiting time of adjuvant treatment had 5-6\% reduced risk of death [comparing to Q1, Q2: HR, 0.95; Q3: HR, 0.94]. Patients received more RT fractions [comparing to 10-29 fractions, 30-33 fractions: HR: 0.62 (biopsy), 0.62 (resection); $\geq 34$ fractions: HR: 0.53 (biopsy), 0.62 (resection)] and high-dose RT [comparing to 34-46 Gy, 50-60 Gy: HR: 0.91 (biopsy), 0.95 (resection); $\geq 60$ Gy: HR: 0.77 (biopsy), 0.88 (resection)] experienced significantly superior survival in both biopsy and resection groups. The impact of timing to adjuvant treatment on GBM survival varied by surgery procedures. Having adjuvant treatment initiated within 21 days for both biopsy and craniotomy groups may not guarantee a significant survival benefit. More RT fractions and high-dose RT are associated with better GBM survival.

53

doi:10.1017/cjn.2018.292

\section{Alterations in the epigenetic profile of glioblastoma tumors within hypoxic tumor regions}

Sheila Mansouri, Carlos Velasquez, Farshad Nassiri, Mark R. Wilson, Suganth Suppiah, Fabio Moraes, Julie Metcalf, Takyee Tung, Olivia Singh, Alireza Mansouri, Kenneth Aldape, Gelareh Zadeh. mansourisheila@gmail.com

Glioblastomas are the most frequent and aggressive primary brain tumor in adults and despite recent therapeutic advances, they are resistant to treatment. Increasing malignancy of gliomas correlates with an increase in cellularity and a poorly organized tumor vasculature, leading to insufficient blood supply, hypoxic areas, and ultimately to the formation of necrosis. Hypoxia induces direct or indirect changes in the biology of solid tumor and their microenvironment through the activation of HIF transcription factors, leading to increased aggressiveness and tumor resistance to therapy. Not much is known about the epigenetic alterations induced by hypoxia and how they could alter tumor biology. In the present study, we have utilized PIMO as a specific marker of hypoxia in glioblastoma patients, treated with PIMO preoperatively. We have estimated PIMO positivity in each tumor $(5-45 \%)$ and determined that it positively correlates with the hypoxia marker CA IX ( $\mathrm{r}=0.57)$. In addition, 10 surgical PIMO cases were dissociated, immune labeled using PIMO antibody, followed by DNA isolation and methylation profiling. Our analysis of differentially top 4000 differentially methylated probes suggests that PIMO-positive (hypoxic) cells are differentially methylated compared to the PIMO-negative cells and these changes are associated with genes involved in hypoxic cellular response. We will validate these findings in additional glioblastoma cases and assess the mechanism of these epigenetic alterations in vitro in glioma stem cell culture conditions and upon exposure of the cells hypoxic conditions.

\section{5-1450 SESSION SEVEN PEDIATRICS}

\author{
13
}

doi:10.1017/cjn.2018.293

The genetic landscape of pediatric low-grade gliomas:
Incidence, prognosis and response to therapy - a SickKids
pLGG Task Force update

Ryall S*, Zapotocky M, Fukuoka K, Guerreiro-Stucklin A, Bennett J. Arnoldo A, Kowalski P, Johnson M, Lassaletta A, Bouffet E, Bartels $U$, Ellison D, Tabori $U, \&$ Hawkins $C$.

scott.ryall@sickkids.ca *BTFC Travel Award Recipient

Molecular characterization of pediatric low-grade glioma (pLGG) over the last decade has identified recurrent alterations, most commonly involving BRAF, and less frequently other pathways including MYB and MYBL1. Many of these molecular markers have been exploited clinically to aid in diagnosis and treatment decisions. However, their frequency and prognostic significance remain unknown. Further, a significant portion of cases do not have any of these alterations and what underlies these cases remains unknown. To address this we compiled a cohort of 562 patients diagnosed at SickKids from 1990-2017. We identified molecular alterations in 454 cases ( $81 \%$ of the cohort). The most frequent events were those involving BRAF; either as fusions (most commonly with KIAA1549 $(30 \%)$ ) or V600E mutations (17\%) and NF-1 (22\%). Less frequently, we identified recurrent FGFR1 fusions and mutations (3\%), MYB/MYBL alterations $(2 \%), \mathrm{H} 3 \mathrm{~F} 3 \mathrm{AK} 27 \mathrm{M}(2 \%)$ or IDH1R132H $(0.5 \%)$ mutations, as well as other novel rare events. Survival analysis revealed significantly better progression-free survival (PFS) and overall survival (OS) of KIAA1549-BRAF fused patients compared to BRAFV600E with 10-year OS $97.7 \%$ (95\%, CI 95.5-100) and $83.9 \%$ (95\%, CI 72.5-95.6), respectively. In addition to survival, molecular alterations predicted differences in response to conventional therapeutics; BRAF fused patients showed a $46 \%$ response-rate, versus only $14 \%$ in V600E patients. pLGGs harboring H3F3AK27M progressed early with median PFS of 11 months. In patients with MYB/MYBL1, FGFR1/FGFR2 alterations, we observed only one death (FGFR1N546K case). The work here represents the largest cohort of pLGGs with molecular profiling and their impact on the clinical behaviour of the disease.

\section{5}

doi:10.1017/cjn.2018.294

CD271/p75NTR is a novel diagnostic marker, prognostic indicator and therapeutic target for SHH medulloblastoma

\section{Liang*, Ludivine Coudière Morrison, Nazanin Tatari, Margaret Stromecki, Agnes Fresnoza, Christopher J. Porter, Marc R. Del Bigio, Cynthia Hawkins Jennifer Chan, Michael D. Taylor, Vijay Ramaswamy, Tamra Werbowetski-Ogilvie. \\ umlian23@myumanitoba.ca * BTFC Travel Award Recipient}

The extensive heterogeneity both between and within the medulloblastoma (MB) subgroups underscores a critical need for variant-specific biomarkers and therapeutic strategies. We 